\title{
Online weight estimation in a robotic gripper arm
}

\author{
Dana COPOT* ${ }^{*}$, Clara IONESCU* ${ }^{*}$, Ioan NASCU ${ }^{\dagger}$ and Robin DE KEYSER* \\ ${ }^{*}$ Ghent University, Department of Electrical energy, Systems and Automation, Research group on Dynamical Systems and Control \\ Technologiepark 914, 2nd floor, 9052 Ghent, Belgium, mail: Dana.Copot@UGent.be \\ †Technical University of Cluj Napoca, Baritiu Street 26-28, 400027 Cluj-Napoca, Romania
}

\begin{abstract}
This paper presents a novel methodology for online, fast and accurate weight estimation technique in a robotic gripper arm. The laboratory setup is inspired from several real life applications of weight estimation in moving cranes, e.g. loading containers in a shipyard, iron scrapping in steel industry, etc. The weight needs to be estimated within a specified time interval and within a tolerance interval for accuracy. The results indicate that the proposed method is suitable for this kind of application and an improvement of $30 \%$ has been achieved compared to the current state of work.
\end{abstract}

Keywords—online estimation, weight measurement, gripper arm

\section{INTRODUCTION}

Cranes are widely used in ports and on large ships for the loading/unloading of containers and bulk materials. Shipboard cranes are widely used to handle and transfer objects from large container ships to smaller lighters or to the quays of the harbours. The control of cranes is always a challenging task which involves many problems such as load sway, positioning accuracy, suppression, collision avoidance, and manipulation security. Therefore, it is important that the load is transported as fast as possible without causing excessive oscillations from one position to the other. The undesirable load oscillations can result in a drop in efficiency, load damages or even accidents. A manifold of control works have been reported in crane control to reduce these oscillations [1], but very little has been done in terms of online weight estimation methods in cranes or in robotic arms [2]-[4].

In order to manipulate objects, contact with the object has to be made and suitable grasping forces have to be applied. For successful manipulation, suitable grasping forces have to be determined without the knowledge of the type of object to be secured by the gripper. Based on grasping operations typically performed by humans, several methods [5]-[7], [8][11] have been proposed for robot grippers.

Designing grippers to perform the grasping of objects with different shape, size, and texture is a complex and expensive task since the manipulating forces cannot be accurately determined. To minimize the overall system complexity and cost, most of the designs rely on feeding the robot with a variety of possible object patterns. The position of the object relative to the gripper, its weight, orientation, and shape are specified beforehand. Using this information, the gripper is guided through a predefined trajectory to reach and to grasp the

This study has been performed in collaboration with Ir. Johan De Vos from Elaut Company, Belgium object with the proper force, while the integrity of the object is guaranteed throughout the manipulation. If the applied force is not sufficient, then the object can slip whereas too much applied force can destroy the object. Moreover, for successful manipulation, the fingers of a gripper have to make contact with the object at the right location and orientation to avoid slippage. One of the problems endured in grasping an object is the ability of the gripper to reach an object in different positions and orientations [11], [12].

In this paper a lab-scale setup has been used to grasp and measure the weight of different objects. Within the current set-up the measurements error can go as high as $40 \%$ and therefore the aim of this study is to reduce this error around $10 \%$. Moreover, the proposed solution should be adaptable to different scenarios. There are several parameters which differ from one set-up to another (e.g. dimensions of the claw, surface type, distance between the claw and the surface, position of the claws, etc).

The paper is structured as follows. In Section II a description of the system is given followed by Section III where the proposed estimation methodology is detailed. In Section IV the results obtained and the discussion are presented followed by conclusions in Section V.

\section{SYSTEM DESCRIPTION}

The gripper movement mechanism is driven by three motors and three main elements:

- $\quad$ first motor is moving the claw up and down and this is depicted by $a$ in figure 1 ;

- $\quad$ second motor is executing the left and right movement of the gripper movement and this is depicted by $b$ in figure 1;

- $\quad$ third motor is performing the forward and backwards movement of the gripper and this is depicted by $c$ in figure 1

- first element (1 in figure 1) is a sustaining platform, this is mechanically connected to the machine and it is used for the forwards and backwards movement;

- $\quad$ second element (2 in figure 1) represents the load cell used to measure the weight of the object;

- third element (3 in figure 1) is the string which connects the claw to the machine. 

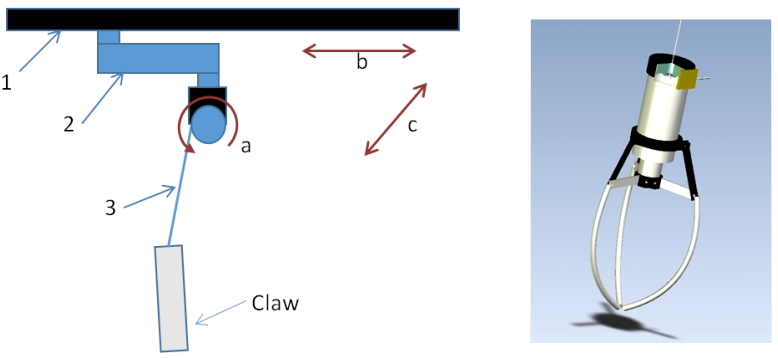

Fig. 1: Left: Movement mechanism of the claw, Right: example of a claw used in claw machine

The most representative parameters for this type of set-up are: weight of the object, type of the load cell and length of the string. A short description of each parameter is given below.

The weight of the object influences the three forces which are applied to the claw during one complete cycle. The weight could be used to identify a certain stage of the cycle (e.g. to detect when the claw has picked an object and in this case the weight will increase or to detect when the object is dropped and in this case the weight will decrease).

The length of the string may be used to analyze the oscillations affecting the weight measurement. The length of the string can be computed using the impulses generated by the motor performing the rotational movement (depicted by $a$ in figure 1). Knowing that the diameter of string role is 34 $\mathrm{mm}$ and that there are 700 impulses/rotation results a length of approximatively $107 \mathrm{~mm}$ with about $1.5 \mathrm{~mm} / \mathrm{impulse}$. A measurement error of about $5 \%$ has been identified as a cause of the string length and this is due to the increase and decrease of the string role diameter during the up and down movement of the gripper.

The load cell is a transducer used to create an electrical signal whose magnitude is directly proportional to the force being measured. On the market exist several types of load cells, such as: strain gauge load cell, hydraulic and pneumatic. This type of load cells are predominantly used in industrial weighing and force measurement due to their consistency and accuracy. An important aspect to be considered is how the measuring cable is affecting the sensitivity of the load cell (a longer cable will cause a greater loss in sensitivity). Another important aspect is the maximum capacity of the load cell. If this is exceeded will result in a deformation of the elastic element and this will cause a shift in the zero point (no load) of the weighing system. The load cell precision used in this study has an accuracy of 1 gram. The signal from the load cell is amplified with a factor of 1000 and it is scaled to a 0-4 interval.

As for every other process there are also disturbances affecting the accuracy of the measurements. For this particular study, the disturbances are caused by: i) the oscillations which appear during lifting; ii) the angle of the gripper when it reaches the target; iii) the shape of the object (this will modify the type oscillations); and iv) the movement of the object in the claw.

Another important aspect is the type of the surface used during measurement. In this paper three types of surfaces have been tested during experiments: i) a soft material (i.e. packing peanuts) generally used for packing fragile object (figure 2 left), ii) small grain like plastic cylinders (figure 2 right) and iii) hard surface, i.e. empty stand surface.

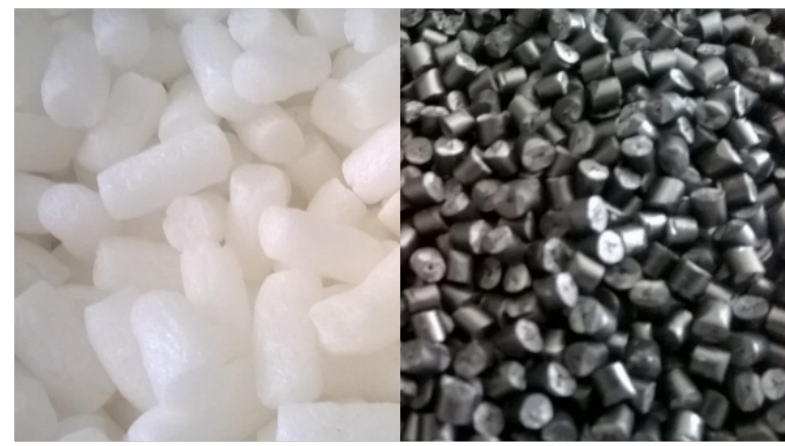

Fig. 2: Left: Soft layer and Right: black grain-like surface.

\section{A. Description of one complete cycle}

A schematic representation of the platform used for experiments is given in figure 3 .

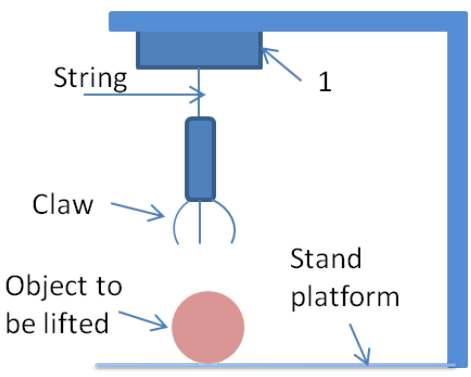

Fig. 3: Schematic representation of the system used for measurements.

The following elements are being depicted: the object to be lifted and the structure sustaining the gripper. The motor performing the rotational movement and the load cell are placed inside box 1 .

In figure 4 a schematic representation of one complete cycle is shown.

1) represents the starting position;

2) is the moment when the start button of the machine has been pressed by the user and the gripper is moving down (at this stage the claws of the gripper are open);

$3)$ is the moment when the gripper reaches the bottom/object, in this step, after a few ms, the claw closes;

4) represents the moment when the object is being lifted;

5) denotes the gripper reached the target and the object is dropped;

6) is the last stage represented by the gripper being in its initial position. 


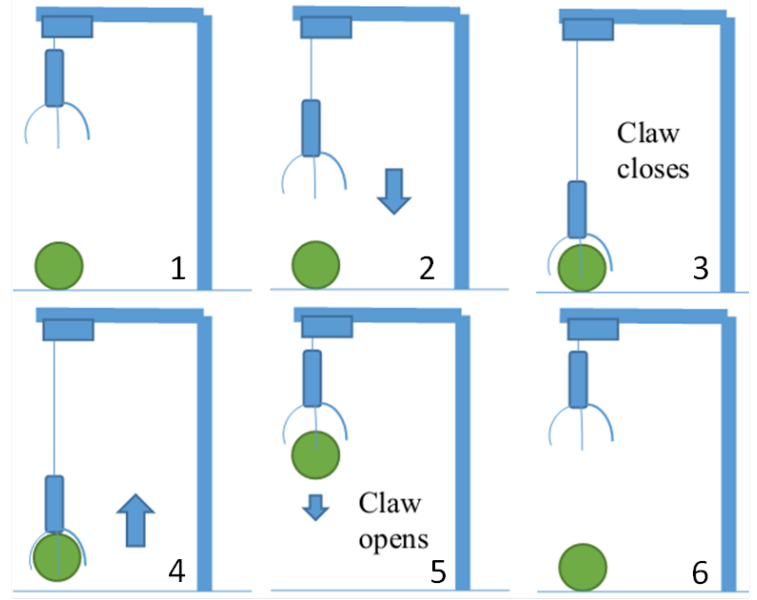

Fig. 4: A pictorial representation of a full cycle.

In figure 5 the output of one complete cycle is presented. For this experiment, the weight of the object used for measurements was approximately 102g. The sampling frequency is 100 samples/second and the output is given in Volts. First part of the signal (flat line) represents the moment before the cycle starts. Once the cycle starts a drop in the signals can be noticed. The moment when the gripper reaches the surface/object is defined by the second drop in the signal. The reason why this occurs is because the weight of the claw is (gradually) no longer sustained by the load cell. When the motor starts pulling up the gripper with the object, the output rises again. This part is highlighted in figure 5 (transient). The signal will increase with the actual value of the object, but disturbances are also present in the signal. Next drop in the signal corresponds to the moment when the gripper arrives up. Then the object is being dropped and the gripper returns to its initial position determining the output to come back to 0 grams.

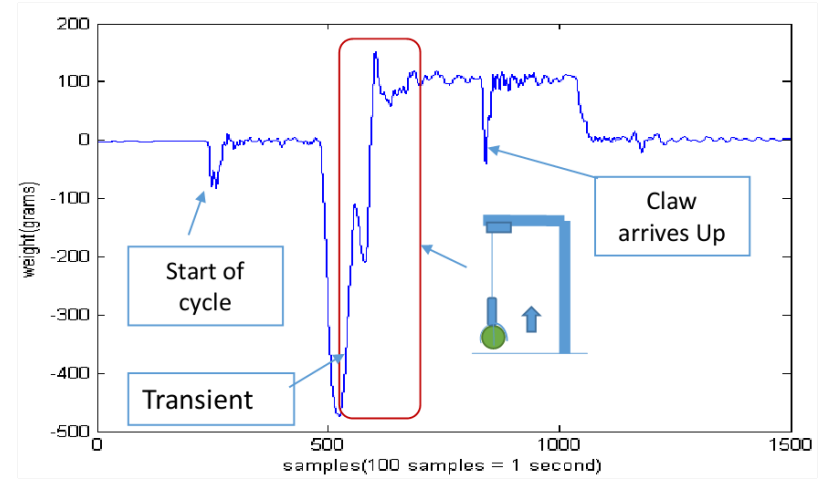

Fig. 5: Output of the load cell for an $102 \mathrm{~g}$ object.

B. Influence of the position of the claw on the surface of the platform on the dynamics of the system

In this subsection the influence of the claw angle on the dynamics of the system is being discussed. For this, several experiments have been performed and we have determined that three different outputs for the transient phase can be distinguished, these are shown in figure 6.

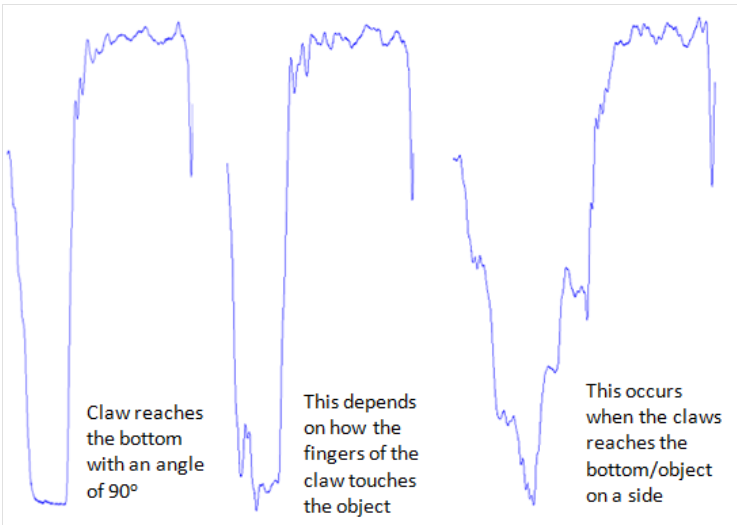

Fig. 6: Outputs load cell depending on the angle of the gripper.

First class of output has been observed when the gripper reaches the bottom/object with an angle of $90^{\circ}$. The dynamics of the system in this case varies as a function of the object shape, height and movement of the object in the claw while it is ascending.

Second class may depend on how the finger of the claw catches the object, e.g.the case when only one finger touches the object and the other two are touching the platform surface and therefore a small angle is being created.

Third class of output occurs when the claw falls on a side. In this case the claw needs a longer time to completely rise from the surface and therefore the time measurements is higher.

\section{PROPOSED ESTIMATION METHODOLOGY}

Since the output of the load cell (used for weight measurements) is in Volts, first step is to make the conversion from Volts to grams using linear regression methods [13].

For this, objects with known weight: 0 grams (empty claw), $33 \mathrm{~g}, 40 \mathrm{~g}, 60 \mathrm{~g}, 100 \mathrm{~g}$ and $140 \mathrm{~g}$ have been used and several experiments were performed for each object. Using the linear regression method we correlated the real weight of the object to the voltage output of the load cell. Linear regression attempts to model the relationship between two variables by fitting a linear equation. One variable is considered to be an explanatory variable, and the other is considered to be a dependent variable. In case of linear regression, predictions of $Y$ are obtained as a function of the independent variable $X$, i.e. $Y_{\text {predicted }}=\alpha X+\beta$. The most common approach for fitting a regression line is the least-square method [13]. The following equation has been used to estimate the parameters:

$$
\alpha=\frac{\sum x_{i} y_{i}-n m_{x} m_{y}}{\sum x_{i}^{2}-n m_{x}^{2}}
$$

where: $x_{i}$ represent the values given to the independent variable $X ; y_{i}$ represent the experimental data, $m_{x}$ is the mean of all $X$ values, $m_{y}$ represent the mean of all data points, $n$ is the number of data points, $\alpha$ is the slope and $\beta$ is the intercept. In order to compute the confidence interval for the 
slope, standard error of the slope $\alpha$ has been calculated using (2).

$$
S_{\alpha}=\frac{s_{\text {est }}}{\sum\left(x_{i}-m_{x}\right)^{2}}
$$

where $s_{\text {est }}$ is computed using (3):

$$
s_{\text {est }}=\sqrt{\frac{\sum\left(Y-Y_{\text {predicted }}\right)^{2}}{n-2}}
$$

Next, the $95 \%$ confidence interval have been calculated:

$$
\begin{aligned}
& \text { Lower bound: } \alpha-t_{95} S_{\alpha} \\
& \text { Upper bound: } \alpha+t_{95} S_{\alpha}
\end{aligned}
$$

These are the basic signal processing techniques used to estimate online the weight of the object grasped by the claw.

\section{A. Identification of the relevant part of the signal which contains the object weight information}

The key part is to detect the most significant segment of the signal to be used for weight measurement. Considering the time constraint (measurement within $0.5 \mathrm{~s}$ ) a specific interval of the measured signal will be used for weight estimation. This is depicted in figure 7 (step 3).

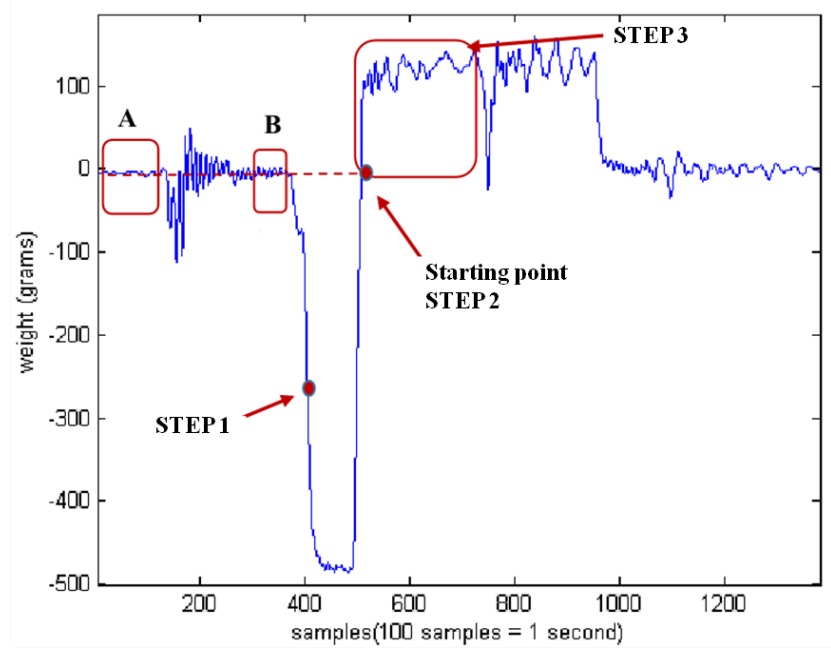

Fig. 7: Graphical illustration of the estimation algorithms related to the data measured online. See text for detailed explanations.

The following algorithm is used for online object weight estimation:

- first, we have to detect when the claw reaches the surface/object. This is denoted by the most significant drop in the signal (step 1).

- $\quad$ second, we go back a number of samples and we take the mean. This mean should correspond to an empty claw (i.e. 0 grams) this is highlighted by zone A (corresponding to the initial state, i.e. before starting a cycle) and B (corresponding with the moment when the gripper start to go down). The part of the signal in between the two zones correspond to the moment when the cycle is started (i.e. when start button is pressed). Several test were performed to identify which zone is best to use to compute the mean value. The obtained results indicated that both zones are suitable, but zone A gives slightly better performance in terms of accuracy. One important aspect is that the part of the signal in between the two zones should not be included in calculation.

- third, using the mean obtained in step 1 we detect/define the measurement starting point (i.e. step 2).

- fourth, now the choice of how far from the staring point we can go in order to obtain the weight of the object within the time constrains. For instance in the case presented in figure 7 the signal corresponding to an interval of approximatively $1.7 \mathrm{~s}$ after the starting point has been considered. However, taking into account the constrains we must detect the weight of the object within $0.5 \mathrm{~s}$. Therefore, the interval $0.5-0.7 \mathrm{~s}$ has been considered to accurately determine the weight of the object (step 3). A close view of the the signal used for estimation is given in figure 8.

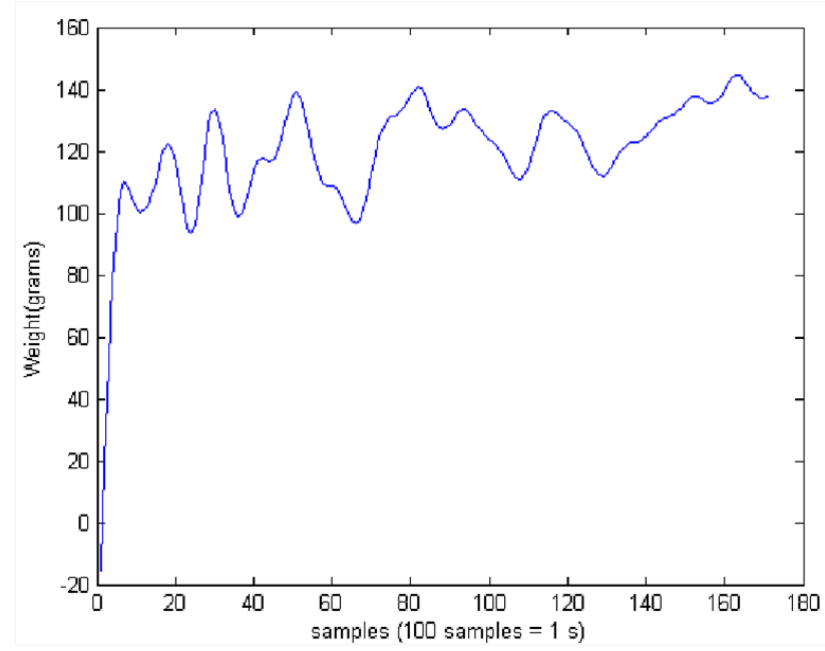

Fig. 8: Part of the signal containing the information about the weight of the lifted object (result of step 4).

\section{RESULTS AND DISCUSSION}

Using the proposed methodology for our data we have: voltage $=\alpha *$ weight $+\beta$, this will indicate how many volts correspond to a known weight. Now, we will have to determine the coefficients for the reverse formula, i.e. weight $=k *$ volts $+q$; where $k=1 / \alpha$ and $\mathrm{q}=-\beta / \alpha$, which will allow us to convert the output signal from volts to grams. Finally, we have obtained the following expression used for calibration: dataInGrams $=625 *$ DataInVolts -498.875 . The $y$ intercept (498.875) represents the weight of the claw. In figure 9 the result is shown.

Next, the results obtained in case of estimating the weight for two datasets (dataset=several experiments for the same object), i.e. $80 \mathrm{~g}$ and $100 \mathrm{~g}$. In figure 10 the results obtained for two sets are presented. From this figure it can be clearly seen that it is hard to make a differentiation between the two 


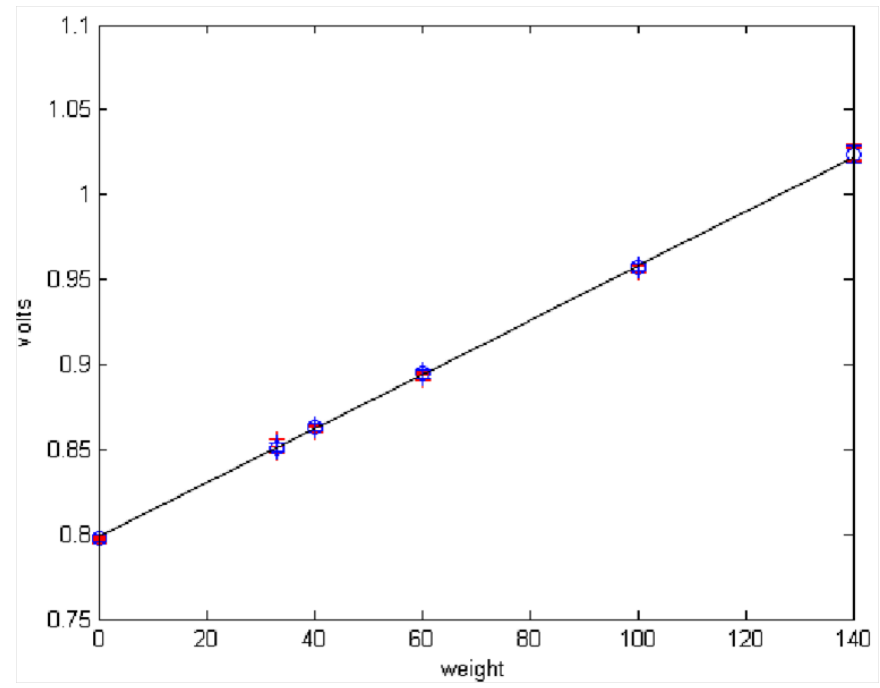

Fig. 9: Calibration results.

sets in the first 0.3-0.4 seconds. To identify the time frame where the differentiation between the sets can be seen we have applied ANOVA technique. More specifically, the mean and the standard deviation for each set has been calculated. If the difference of the means for each set is higher that the standard deviation of each set then we can clearly differentiate between them. The algorithm is presented in detail in [14]. The results obtained after applying the ANOVA analysis are presented in figure 11 .

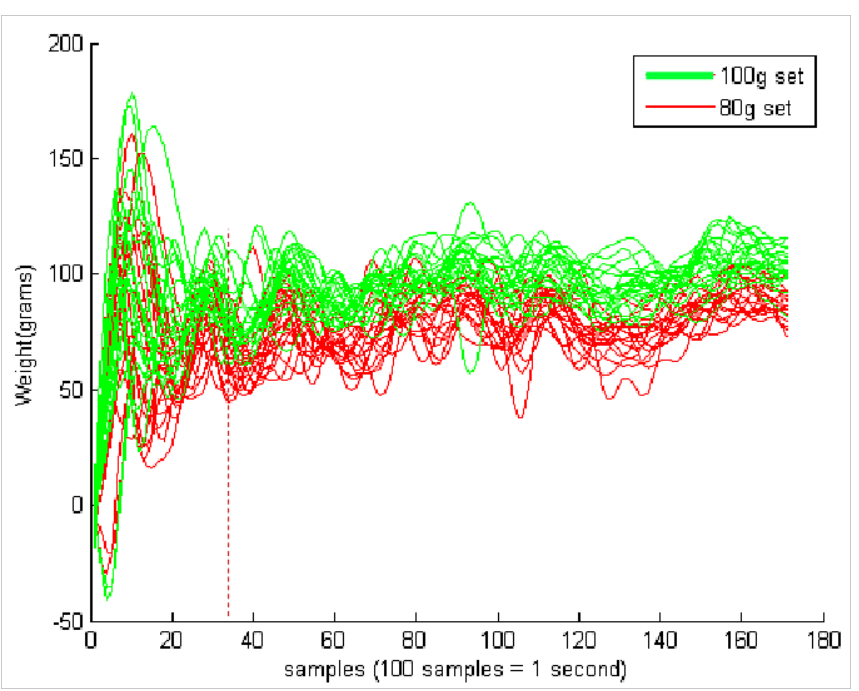

Fig. 10: Sets of measurements for $100 \mathrm{~g}$ and for $80 \mathrm{~g}$ objects. Observe the difference. See text for explanations.

From figure 11 we can conclude that the difference of the means becomes higher than the standard deviation only after 0.3 seconds. After several tests, the results suggest that for most of the cases the sets can be differentiated around 0.4 seconds. Based on the acquired knowledge, we have decided to use the mean of the data between 0.4-0.6 seconds (samples 4060 ). This value is automatically identified (for each individual experiment) and is further used in a weighted linear regression

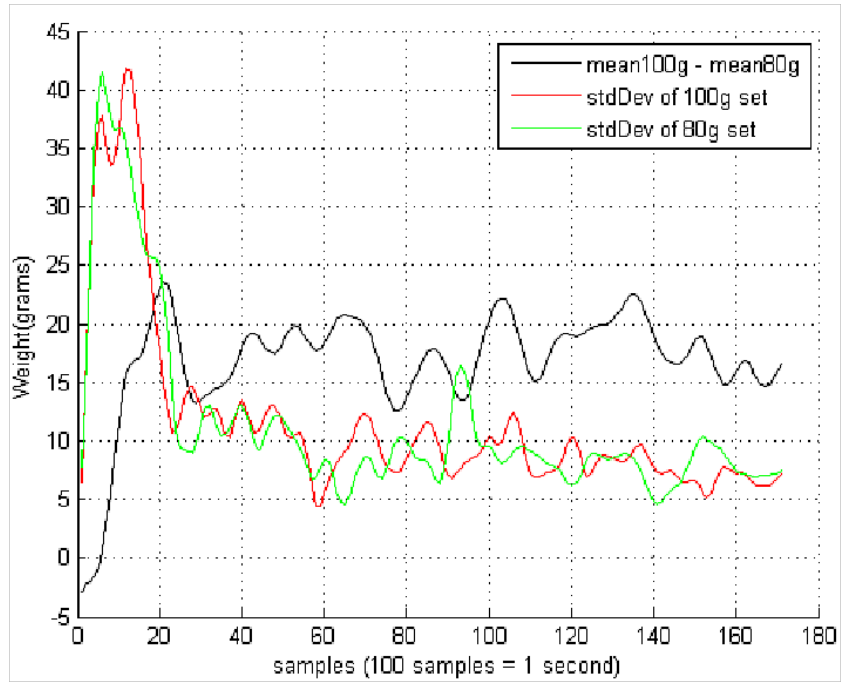

Fig. 11: Results obtained after ANOVA method has been applied to the datatsets.

analysis in order to obtain a model for weight prediction. In figure 12 the results for the estimated and the real weight for several object are shown. It can be noticed that the data points varies more than in the case of the tests done for calibration. This is due to the disturbances (e.g. position of the claw when it arrives on the surface, the shape of the object, movement of the object in the claw, etc.).

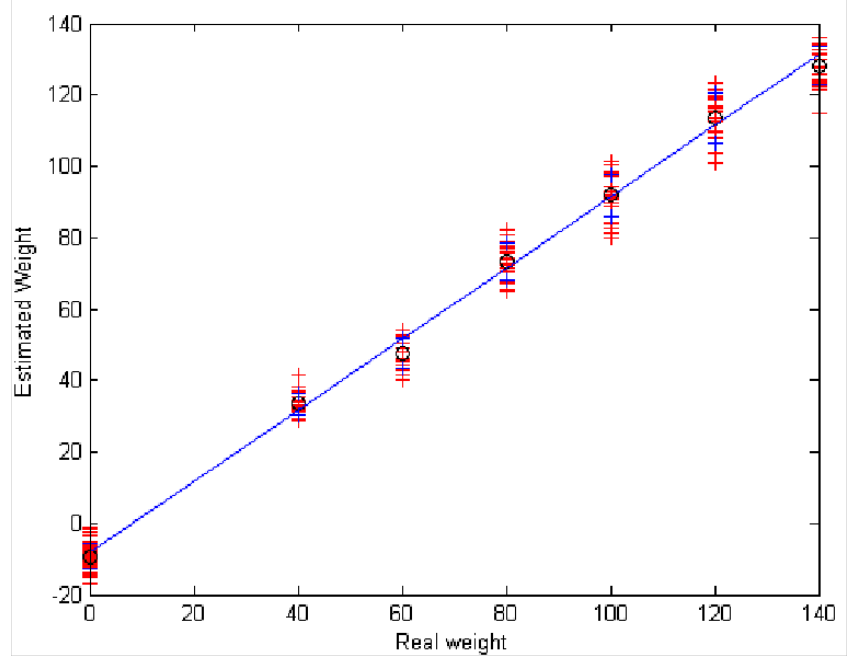

Fig. 12: Estimated vs real weight of the object.

In figure 12 the estimated weight versus the real weight of the object is represented. The red + represent the means for each individual measurement, blue + represent the means of each group \pm the standard deviation of each group and $o$ represent the means of each group.

A total number of more than 200 experiments have been performed for objects of different weights. The results obtained for different setups (i.e. different surfaces) and for different weights are summarized in the tables below. As it can be no- 
ticed from the tables below the online estimation methodology gives better results than the actual method implemented in practice, i.e. the measurement error has been reduced from more than $40 \%$ while with the method proposed in this paper the error has been reduced to less than $15 \%$.

\begin{tabular}{|r|c|c|c|}
\hline Errors & $\begin{array}{c}\text { abs(error) } \leq \mathbf{1 0 g} \\
\text { no. of tests [\%] }\end{array}$ & $\begin{array}{c}\text { abs(error) } \leq \mathbf{1 5 g} \\
\text { no. of tests [\%] }\end{array}$ & $\begin{array}{c}\text { abs(error) }>\mathbf{1 5 g} \\
\text { no. of tests [\%] }\end{array}$ \\
\hline $\begin{array}{r}\text { 58 grams toy } \\
\text { 102 grams toy }\end{array}$ & $94 \%$ & $100 \%$ & $0 \%$ \\
\hline 130 grams toy & $83 \%$ & $94 \%$ & $6 \%$ \\
\hline 163 grams toy & $95 \%$ & $95 \%$ & $5 \%$ \\
\hline gran & $100 \%$ & $0 \%$ \\
\hline
\end{tabular}

Table 1: Results obtained for soft surface (thin layer).

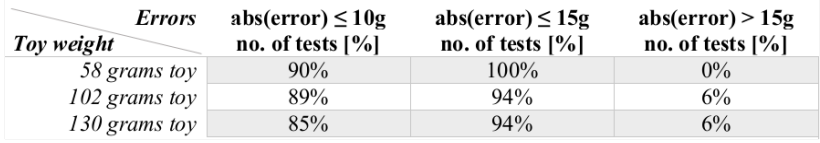

Table 2: Results obtained for soft surface (thick layer).

\begin{tabular}{|c|c|c|c|}
\hline $\begin{array}{l}\text { Errors } \\
\text { Toy weight }\end{array}$ & $\begin{array}{c}\text { abs(error) } \leq 10 \mathrm{~g} \\
\text { no. of tests }[\%]\end{array}$ & $\begin{array}{c}\operatorname{abs}(\text { error }) \leq 15 \mathrm{~g} \\
\text { no. of tests }[\%]\end{array}$ & $\begin{array}{c}\text { abs }(\text { error })>15 \mathrm{~g} \\
\text { no. of tests [\%] }\end{array}$ \\
\hline 36 grams toy & $85 \%$ & $100 \%$ & $0 \%$ \\
\hline 58 grams toy & $84 \%$ & $100 \%$ & $0 \%$ \\
\hline 102 grams toy & $90 \%$ & $100 \%$ & $0 \%$ \\
\hline 130 grams toy & $80 \%$ & $85 \%$ & $15 \%$ \\
\hline
\end{tabular}

Table 4: Results obtained for black surface.

\begin{tabular}{|r|c|c|c|}
\hline Eoy weight & $\begin{array}{c}\text { Ebs(error) } \leq \mathbf{1 0 g} \\
\text { no. of tests [\%] }\end{array}$ & $\begin{array}{c}\text { abs(error) } \leq \mathbf{1 5 g} \\
\text { no. of tests [\%] }\end{array}$ & $\begin{array}{c}\text { abs(error) }>\mathbf{1 5 g} \\
\text { no. of tests [\%] }\end{array}$ \\
\hline 36 grams toy & $100 \%$ & $100 \%$ & $0 \%$ \\
\hline 58 grams toy & $85 \%$ & $100 \%$ & $0 \%$ \\
\hline 102 grams toy & $90 \%$ & $100 \%$ & $0 \%$ \\
\hline 130 grams toy & $70 \%$ & $90 \%$ & $10 \%$ \\
\hline
\end{tabular}

Table 3: Results obtained for hard surface (empty).

\section{CONCLUSiOnS}

In this paper a novel methodology for online estimation of weight in a robotic gripper arm has been presented. The proposed methodology shows good performance and the error has been improved from 40 grams (for weights between $30 \mathrm{~g}$ - $150 \mathrm{~g}$ ) to errors smaller or equal to 15 grams (i.e. an improvement of $66 \%$ ). The aim of this study was to reduce the error but at the same time to obtain the results in about $0.5 \mathrm{~s}$. However, to fulfill both objectives a trade off has to be made. Therefore, for all th experiments a accurate measurement can be obtained in a time interval between $0.5 \mathrm{~s}-0.7 \mathrm{~s}$.

\section{REFERENCES}

[1] M. Kenison, W. Singhose, Input shaper design for double-pendulum planar gantry cranes, in Proc. IEEE Conf. Control Appl., 1999, pp. 539 544.

[2] Q. Ngo, K. Hong, Sliding-mode antisway control of an offshore container crane, IEEE/ASME Trans. Mechatronics, 17(2), pp. 201-209, 2012.

[3] N. Sun, Y. Fang, New energy analytical results for the regulation of underactuated overhead cranes: An end-effector motion-based approach, IEEE Trans. Ind. Electron., 59(12), 4723-4734, 2012.
[4] E. Delgado, M. Daz-Cacho, D. Bustelo, A. Barreiro, Generic ap-proach to stability under time-varying delay in teleoperation: Application to the position-error control of a gantry crane, IEEE/ASME Trans. Mechatronics, 18(5), 1581-1591, 2013.

[5] A. Anglani, F. Taurisano, R. De Giuseppe, C. Distante, Learning to grasp by using visual information, Proc. IEEE Conf. Automation,. 714, 1999.

[6] D. M. Gorinevsky, A. M. Formalsky, A. Y. Schneider, Force Control of Robotics Systems. Boca Raton, FL: CRC Press LLC, 1997.

[7] E. Kafalea, E. Mael, R. P. Wurtz, An integrated object representation for recognition and grasping, Proc. 3rd Int. Conf. Knowledge-Based Intelligent Information Engineering Information Systems, 423-426, 1999.

[8] T. Maeno, S. Hiromitsu, T. Kawai, Control of grasping force by detecting stick/slip distribution at the curved surface of an elastic finger, Proc. IEEE Int. Conf. Robotics and Automation, 3895-3900, 2000.

[9] A. Moon, M. Farsi, Grasp quality measures in the control of dextrous robot hands, Proc. Inst. Elec. Eng., 1-4, 1996.

[10] A. Rovetta, X. Wen, Conceptual design of a hand controller with sensors integration, Proc. IEEE Int. Workshop on Intelligent Motion Control, Istanbul, Turkey, 383-388, 1990.

[11] J. Su and Y. Xi, Path planning for robot hand/eye system to intercept moving objects, Proc. IEEE Conf. Decision and Control, 2963-2968, 2000.

[12] E. Kafalea, E. Mael, and R. P. Wurtz, An integrated object representation for recognition and grasping, Proc. 3rd Int. Conf. KnowledgeBased Intelligent Information Engineering Information Systems, 423426, 1999.

[13] D.C. Montgomery,E.A. Peck, Introduction to Linear Regression Analysis, 4th edition. John Wiley and Sons, Ltd., 2007.

[14] R.M. Mickey, O.J Dunn, V.A. Clark, Applied Statistics: Analysis of Variance and Regression, 3rd Edition, ISBN: 978-0-471-37038-3, 2004. 